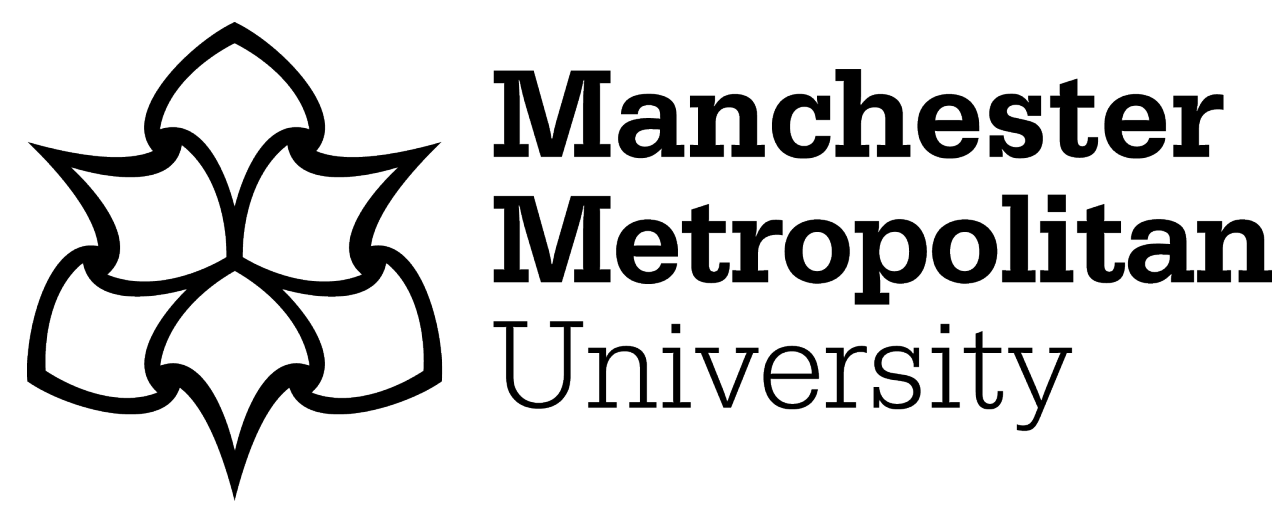

Iwendi, C, Maddikunta, PKR, Gadekallu, TR, Lakshmanna, K, Bashir, AK and Piran, MJ (2021) A metaheuristic optimization approach for energy efficiency in the loT networks. Software - Practice and Experience, 51 (12). pp. 25582571. ISSN 0038-0644

Downloaded from: https://e-space.mmu.ac.uk/625907/

Version: Accepted Version

Publisher: Wiley

DOI: https://doi.org/10.1002/spe.2797

Please cite the published version 


\title{
A Metaheuristic Optimization Approach for Energy Efficiency in the IoT Networks
}

\author{
Celestine Iwendi ${ }^{1}$, Praveen Kumar Reddy $\mathbf{M}^{2, *}$, Thippa Reddy $\mathbf{G}^{3, *}$, Kuruva \\ Lakshmanna $^{4}$, Ali Kashif Bashir ${ }^{5, *}$, MD. Jalil Piran ${ }^{6}$
}

${ }^{1}$ Department of Electronics, BCC of Central South University of Forestry and Technology, Changsha, 410004 China; celestine.iwendi@ieee.org

${ }^{2}$ School of Information Technology and Engineering, Vellore Institute of Technology, India; praveenkumarreddy@vit.ac.in

${ }^{3}$ School of Information Technology and Engineering, Vellore Institute of Technology, India; thippareddy.g@vit.ac.in

${ }^{4}$ School of Information Technology and Engineering, Vellore Institute of Technology, India; lakshman.kuruva@vit.ac.in

${ }^{5}$ Department of Computing and Mathematics Manchester Metropolitan University Manchester, M15 6H U.K.; dr.alikashif.b@ieee.org

${ }^{6}$ Computer Engineering Department, Sejong University, Seoul, South Korea; piran@sejong.ac.kr

\section{* Correspondence: praveenkumarreddy@vit.ac.in; thippareddy.g@vit.ac.in; dr.alikashif.b@,ieee.org}

Abstract: Recently Internet of Things (IoT) is being used in several fields like smart city, agriculture, weather forecasting, smart grids, waste management, etc. Even though IoT has huge potential in several applications, there are some areas for improvement. In the current work, we have concentrated on minimizing the energy consumption of sensors in the IoT network that will lead to an increase in the network lifetime. In this work, to optimize the energy consumption, most appropriate Cluster Head $(\mathrm{CH})$ is chosen in the IoT network. The proposed work makes use of a hybrid meta-heuristic algorithm, namely, Whale Optimization Algorithm (WOA) with Simulated Annealing (SA). To select the optimal CH in the clusters of IoT network, several performance metrics such as the number of alive nodes, load, temperature, residual energy, cost function has been used. The proposed approach is then compared with several state-of-the-art optimization algorithms like Artificial Bee Colony (ABC) algorithm, Genetic Algorithm (GA), Adaptive Gravitational Search algorithm (AGSA), Whale Optimization Algorithm (WOA). The results prove the superiority of the proposed hybrid approach over existing approaches.

Keywords: Whale Optimization; Simulated Annealing; Hybrid Algorithms; Cluster Head; Internet of Things; Wireless Sensor Networks; Meta-Heuristic Algorithms.

\section{Introduction}

Internet of Things (IoT) is a system that can be the interconnection of anything that can transmit and receive data, which may be wireless sensor networks (WSN), robots, smartwatches, humans, animals, plants, vehicles, etc. Every component in IoT should have a unique identifier. Over the past decade, the usage of IoT has grown tremendously throughout the world. Some of the prominent applications of IoT are in the building of smart cities, Home Surveillance systems, farming, healthcare systems, etc. [1]. Although IoT is used in many applications, the success of these applications depends on solving many challenges. Some of 
the challenges of successful implementation of IoT [2], [3] are Integration of several enabling technologies and standards that deal with storage, sensing, computational capabilities, connectivity, Traffic models that deal with data transfer across several components in IoT system and their load balancing, Standardization, Availability, Reliability, Performances, and Quality of Services (QoS), Self-configuration and management of several components in IoT system, Unique identification, Energy optimization, Security and privacy, Environmental issues.

This paper focusses on addressing the energy optimization research challenge for wireless sensors in IoT. As the wireless sensors in IoT systems continuously sense and generate a huge amount of data, the system consumes enormous power. This may result in a reduction of the lifetime of sensor devices, sustainability of the network. In order to solve this issue, we have to optimize the power/energy consumption by WSN sensors [4]. In the IoT system, there will be several wireless sensor nodes. All these nodes try to communicate with the IoT base station (BT), which may lead to overloading of the BT, transferring of redundant data [5]. To overcome this drawback, a group of nodes are divided into several clusters and a $\mathrm{CH}$ has to be chosen for every cluster. Data is sent to the $\mathrm{CH}$ by all other nodes in the cluster. Then every $\mathrm{CH}$ will consolidate the data and send the same to the IoT BT. In each cluster, $\mathrm{CHs}$ are selected based on various performance metrics like temperature, load, distance, residual energy from $\mathrm{CH}$ to the IoT BT, delay. In this paper to address the energy optimization of WSN sensors, a hybrid model that integrates whale optimization technique with simulated annealing (WSA) is used. The primary motivation behind this work is optimizing the energy consumption of the WSN sensors in the IoT system which leads to increased IoT system lifespan, network efficiency with the IoT system. Figure 1 illustrates the IoT based WSN architecture.

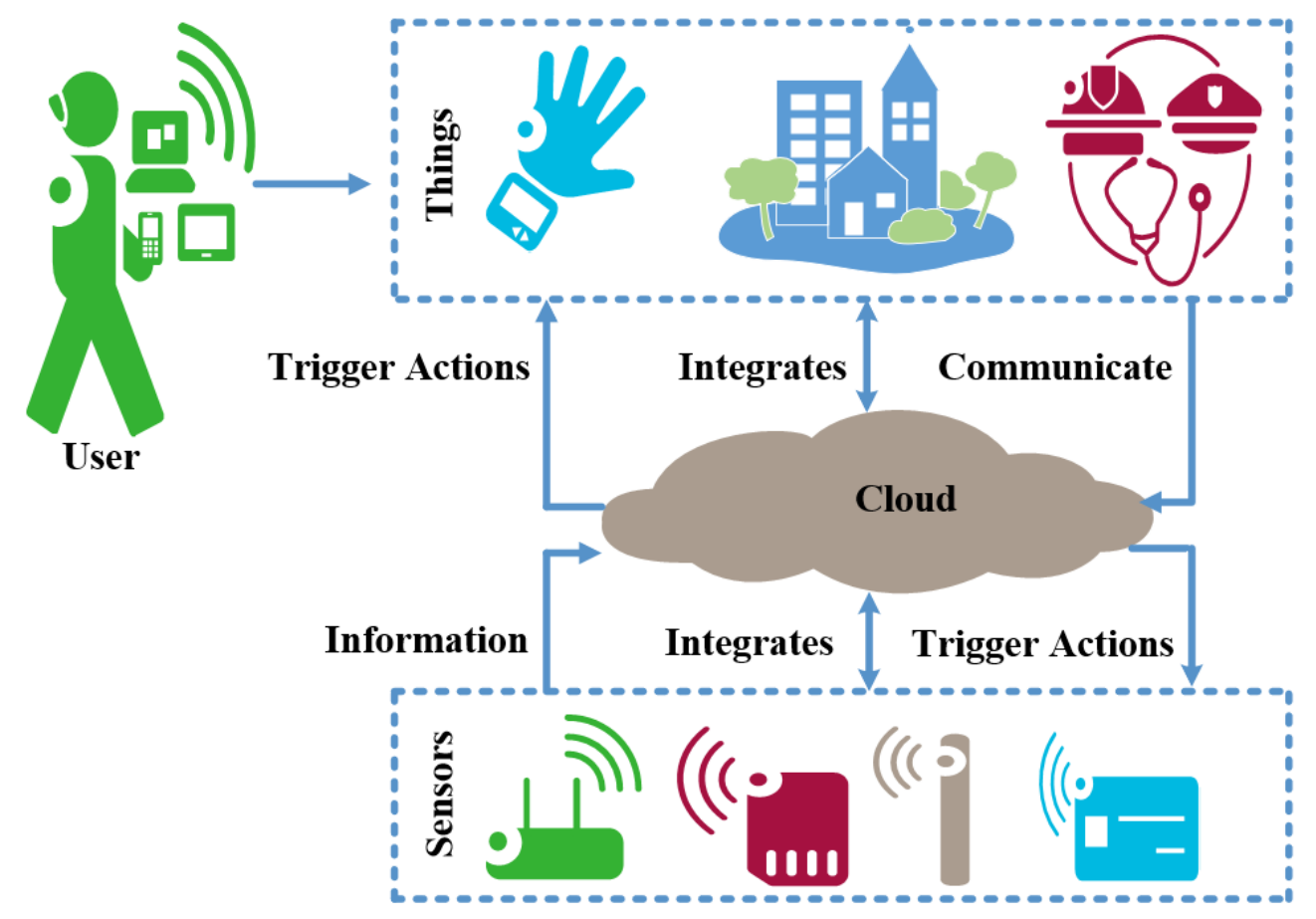

Figure 1: IoT Architecture

Clustering plays a vital role in preserving energy in IoT networks by delegating the responsibility of communication overhead to the CHs. In the earlier models, distributed clusters were introduced in which; all sensor nodes determine functions and transmissions [6]. The varied cluster size becomes a vital issue in this model, such as high-node clusters 
and low node clusters, which significantly reduces the performance in terms of energy because the distance among the BS and CHs varies too long for some nodes. However, it suffers from high computational time, which leads to delay and load. The former algorithms fail due to high energy consumption [7]. With this intention, various metaheuristic and evolutionary optimization models were implemented to save energy. All such algorithms focused on the continuous nodes and let others sleep to minimize energy consumption. However, IoT consumes more energy due to various problems such as data-traffic, load, and temperature [8]. Thus, there exists a necessity to propose an energy-efficient IoT framework such that energy is normalized, thereby achieve network stability and prolonged lifetime. Towards this goal, a hybrid Whale Optimization Algorithm- Simulated Annealing (WOA$\mathrm{SA}$ ) is used for choosing optimal $\mathrm{CH}$ in an IoT network in this work. This hybrid algorithm makes use of SA to enhance the exploitation by searching the most prominent regions identified by the WOA algorithm.

The main contributions of the proposed hybrid model are:

1) Selection of best cluster head using the WSA hybrid model.

2) Optimization of performance metrics of WSN sensors that affect the energy consumption like load, temperature, delay, the distance between the base station to the cluster head using the WSA model.

The remaining sections are arranged as follows: Section 2 discusses the recent literature about energy optimization of WSN sensors in the IoT system is discussed. The background details related to the proposed architecture are discussed in section 3. In section 4, a detailed discussion of the proposed architecture is illustrated. In section 5, the experimental result analysis. In section 6 , conclusion and future work are discussed.

\section{Literature Survey}

Several works have been carried out to improve the lifetime of IoT networks. A survey of some of the prominent works has been explored in this section. In [9], the authors suggested a Cooperative Multiple-Input-Multiple-Output (CMIMO) increase IoT-based network's lifetime, using a metaheuristic algorithm called the Quantum Inspired Particle optimization (QPSO). QPSO aims to dynamically choose the best cooperative MIMO transmitter and receiver devices for each hop, resulting in increased network life. In this work, the authors have adopted additive white Gaussian noise (AWGN) for intracluster communication. Experimental results show that the authors used three hops routing, a simulation was performed to check the battery life of the network for only $2 * 2 \mathrm{MIMO}$ and $3 * 3 \mathrm{MIMO}$.

In [10], the authors propose a neuro-fuzzy rule clustering approach to improve end-to-end performance and effective packet delivery ratio. This algorithm is intended to develop the best cluster head and route packets effectively, which would increase network life. Network performance is accessed by calculating cluster head residual energy and cluster head to sink node distance. The simulation results indicate that the use of a neuro-fuzzy system minimizes energy utilization and maximizes network life. The authors here have concluded that all network nodes are trustful, which is not always true.

In [11], the authors suggest a protocol for selecting the best cluster header. As sensor nodes have less battery life, there is less memory and a poor range of communication. The authors used this proposed communication protocol to transmit the information received to IoT BT. During the cluster head selection process, two best cluster headers are chosen in the same cluster to increase the sustainability of the network. The process for choosing dual cluster 
heads is an information fusion of data entropy. This data about entropy is then used for classification and fusion, data transmission and fusion of classification results. The experimental analysis concludes that the proposed protocol for selecting the best dual cluster heads enhances network life, throughput.

In [12], the authors propose a hybrid of Ant Lion Optimization (ALO) and Moth Flame Optimization to choose the best CH in the WSN -IoT network. The goal of this model was to select the best $\mathrm{CH}$, selecting the node that is less far from a base station and takes less time to transmit the data, preserving the energy of the node, and balancing IoT devices load and temperature. The hybrid model was compared to conventional models such as ABC, PSO, GA, ALO, GSA, AGSA and MFO, respectively. The new hybrid model extends the life expectancy of the WSN-IoT network by retaining more energy.

In [13], the authors implement Self Adaptive Whale Optimization Algorithm (SAWOA) to develop the energy-aware $\mathrm{CH}$ selection model for the WSN-IoT network. In order to choose the best $\mathrm{CH}$ performance metrics such as delay, time, power, load and temperature were considered. The proposed model is compared with several algorithms like ABC, PSO, GSA, genetic algorithm, adaptive GSA and CH selection models based on WOA. The results of the simulation model demonstrate that SAWOA is successful in selecting a cluster head so that network life is prolonged.

In [14], the authors use a novel MOFGSA algorithm for choosing the best CH. Every node's energy in IoT initially supplies the packets considerably for efficient routing. This FGSA algorithm is a hybrid of fractional theory and GSA. Performance is compared with existing algorithms such as ABC, GSA and Multiparticle Swarm Cooperative Algorithm and ensures that the lifetime of IoT nodes is extended.

In [15], the authors propose a HEEQA algorithm for achieving a balance between the energy of devices. The parameters of MAC layer then set to minimize energy utilization in the systems. Achieving QoS is a major challenge in IoT devices and energy balance is important to prolong the life of the sensors. This is achieved by combining quantum particle swarm optimization (QPSO) with advanced non controlled genetic sorting algorithm (NGSA). The simulation result has shown that the HEEQA algorithm optimizes energy consumption, hence optimizing network life, throughput, and supply.

In [16], the authors propose an uneven clustering of the time-delay routing protocol to address the issue of consumption of energy which is not balanced and data transmission that is delayed in WSNs. It is compared with various existing algorithms. The results of simulated experiments showed that this work improves network performance and balances the energy use of the network, extending network life. The algorithm suggested is particularly suitable for IoT applications with minimal delay.

In [17], the authors designed a multimodal data correlation algorithm, which includes data correlation analysis and device clustering, increasing the cognitive data capacity of CIoT. Firstly, a DMDC model is constructed based on CCA to transform the data functions and evaluate the data connection. The DMDC framework was used to evaluate the connected information. Correlation analysis is then proposed to categorize the system using a heterogeneous clustering model (HDC).

In [18], the authors introduce a dynamic IoT Data Stream adaptive clustering method to determine various classes found in the data stream depending on data distribution. The results 
of the proposed clustering technique can be used as an input for pattern, event recognition and real data streaming analysis.

In [19], the authors introduce a novel method, NOMA in connection with fog nodes with IoT devices, considering IoT device clustering and power allocation in Hybrid NOMA systems. Complex clustering of IoT devices is addressed in the first phases in order to reduce network complexity and the lag of improved channel conditions for IoT devices. In the second phase, energy management based on power allocation is solved through the Nash bargaining solution (NBS). The results of the simulation show that the proposed system can simultaneously achieve higher spectrum efficiency and ensure equality between other schemes and IoT devices.

In [20], the authors propose a Data Clustering System for Privacy and Availability (PADC) that enhances the collection and distance calculation method of the initial central points from one central point to another based on the k-mean algorithms and differential data privacy. The PADC system adds to each cluster weights by the density of the cluster in every iteration, thus helping to calculate the relative distance between data points for more exact division results. Evaluation of performance shows that the proposed scheme improves the availability of clustering results at the same level of privacy compared to existing differential algorithms in terms of privacy, which suggests that the proposed PADC scheme outperforms other intelligent IoT electric service systems.

In [21], the authors propose a Hy-IoT algorithm that allows control of the clustering to include the area of real-world cyber IoT architecture. The weighable election probability is updated on the basis of residual power, distances, and existing heterogeneity conditions to make use of the $\mathrm{CH}$ area by examining different and dynamic measures. The simulation shows Hy-IoT extends the lifetime of the network and increases output compared with SEP, LEACH, and Z-SEP.

In [22], the authors proposed a novel OGMAD method is proposed which adapts the superframe in the active period with respect to the requested data. This proposed method improves the utilization of links and also incorporate more GTS nodes. In [23], the authors propose an encryption protocol based on context awareness for IoT network which selects the best encryption protocol based on data confidentiality and device specifications. This method saved memory consumption by $79 \%$, battery consumption by $56 \%$ and reduced execution time by $68 \%$.

In [24], the authors propose a sensed environment based robot design using IoT devices through which robots can communicate with any device in the IoT network. In [25], the author's sensor routing protocols in several applications. In [26], the authors proposed a neuro-fuzzy approach for intrusion detection in energy-efficient WSN networks. In [27], the authors propose a framework that employs data mining in clouds, which can be extended to IoT networks to detect and control attacks in a cloud environment. In [28] and [29], the authors propose a novel and innovative mechanism based on IoT network to improve surveillance at homes using a mobile app and web application. This approach makes sure that sufficient security is given to the users to detect and prevent burglaries at their homes.

From the above discussion, it can be concluded that even though there are many cluster head selection models available, they suffer from high energy consumption. To address this issue, a hybrid WOA-SA algorithm is used to optimize energy consumption by choosing optimal $\mathrm{CH}$ in the IoT network. 


\section{IoT-based WSN adaptive cluster head selection}

IoT network consists of multiple sensor nodes with minimal storage and huge power consumption [30-34]. As these nodes continuously generate data, there will be more use of the battery. If energy consumption is more network's lifetime is reduced. Choosing the best $\mathrm{CH}$ is one of the techniques for optimizing energy. Clustering is a mechanism to divide the sensor nodes into groups by selecting the leader of each group based on various parameters. Groups are referred to as clusters, and group leaders are referred to as CHs. Clustering helps to optimize network resources utilization, management of node failures, load balancing, energy consumption, and network life management. Some of the parameters used to select the best cluster head include communication costs within the cluster, energy of the sensors, location of the node from the BT. After selecting the best $\mathrm{CH}$, the cluster nodes will transfer the data to their $\mathrm{CH}$. $\mathrm{CH}$ will forward the consolidated data to central BT. In this work, we are considering $\mathrm{C}$ no of clusters denoted as $\mathrm{C}_{\mathrm{i}}$, where, $\mathrm{i}=1,2, \ldots, \mathrm{N}$. $\mathrm{X}$ no of nodes in each cluster denoted as $\mathrm{Xi}$, where, $\mathrm{i}=1,2, \ldots, \mathrm{M}$. Total no of $\mathrm{CH}$ are denoted as $\mathrm{T}_{\mathrm{CH}}$. Only the chosen $\mathrm{T}_{\mathrm{CH}}$ can communicate with the IoT base satiation. Choosing the best cluster head has now become a challenging task for IoT based WSN that leads to an increase in network life.

\subsection{Fitness function}

In traditional WSN, $\mathrm{CH}$ is chosen based on parameters such as distance, delay, energy. Since we are integrating WSN with IoT, it is essential to work on other parameters such as load and temperature. Thus, to improve network performance, network lifetime, the $\mathrm{CH}$ is chosen based on a node with high energy and less load, delay, distance, and temperature. To improve the stability and performance of the network, the fitness function should be maximized which is represented in Equation 1.

$$
\mathbf{F F}_{\mathbf{i}}=\alpha * \mathbf{F F}_{\text {Temperature }}+\boldsymbol{\beta} * \mathbf{F F}_{\text {Load }}+\vartheta \mathbf{F F}_{\text {Energy }}+\boldsymbol{\varphi}\left(1-\mathbf{F F}_{\text {Distance }}\right)+\boldsymbol{\omega}\left(1-\mathbf{F F}_{\text {Delay }}\right)
$$

Where $\alpha, \beta, \varphi, \varpi, \vartheta$ the weighted parameters, unity are should be the summation of these values.

The mathematically modeled estimation of the five parameters defined in this experiment, as explained in the following subsections.

\subsubsection{Energy Computation}

The energy consumed in a single path in IoT systems can be split into two components. The first component is the energy consumed by all power amplifiers EPA, the second component is the energy consumed by remaining circuit blocks, EC. The total energy consumption, E for every link is given as follows:

$$
E=E P A+E C
$$

The energy in IoT nodes cannot be recharged. Initially, the energy of the IoT node is $\mathrm{E}_{0}$. Each node in a cluster has to transfer the packet to the $\mathrm{CH}$. During transmitting of packets from $\mathrm{x}^{\text {th }}$ node to $\mathrm{y}^{\text {th }} \mathrm{CH}$ there is a loss of energy in both $\mathrm{CH}$ and node. Every IoT node has the hardware for the receiver and transmitter. Whenever a node transfers or receives data, the energy dissipates in the form of a transmitter and receiver. Energy dissipation in the transmitter is a form of power amplifier, radio electronics, where the energy dissipation in the receiver is from radio electronics. There are two ways to dissipate energy, the first of which is when the node transfers $\mathrm{K}$ bytes of data to the $\mathrm{CH}$ given in Equation 3. Equation 5 shows the energy dissipation when the $\mathrm{CH}$ receives $\mathrm{K}$ bytes of data from the normal node. 
$E\left(D_{N}^{a}\right)=\left(E_{e} * k\right)+\left(E_{f s} * k\right) *\left\|D_{N}^{a}-D_{C H}^{n}\right\|$

$\boldsymbol{E}\left(\boldsymbol{D}_{N}^{a}\right)$ is energy dissipation of a ${ }^{\text {th }}$ normal node, $\boldsymbol{E}_{\boldsymbol{e}}$ is electronic energy, $\boldsymbol{E}_{f s}$ is free energy space, $\boldsymbol{D}_{N}^{a}-\boldsymbol{D}_{\boldsymbol{C H}}^{n}$ the distance between $\mathrm{a}^{\text {th }}$ normal node to $\mathrm{nth} \mathrm{CH}$.

$E_{e}=E_{T}+E_{D A}$

$E_{T}$ is transmitter energy and $E_{D A}$ is data aggregation energy.

$E\left(D_{C H}^{n}\right)=E_{e}^{*} k$

After transmitting and receiving the data, the energy must be updated in both the normal node and the $\mathrm{CH}$. The updated energy available in the normal node after transmitting the data to the $\mathrm{CH}$ is given in Equation 6. The updated energy available in $\mathrm{CH}$ after receiving the data from the normal node is given in Equation 7. The normal node transfers the data to the $\mathrm{CH}$ till the energy of the node is not zero. The node is called a dead node if the energy becomes 0. The fitness function for energy is expressed in Equation 8. In order to choose the best $\mathrm{CH}$ one of the performance metrics is energy. Where the energy of the node should be high to select as $\mathrm{CH}$.

$$
\begin{aligned}
& E_{A+1}\left(D_{N}^{a}\right)=E_{A}\left(D_{N}^{a}\right)-E\left(D_{N}^{a}\right) \\
& E_{A+1}\left(D_{C H}^{n}\right)=E_{A}\left(D_{C H}^{n}\right)-E\left(D_{C H}^{n}\right) \\
& F F_{\text {Energy }}=\frac{1}{X}\left\{\sum_{a=1}^{X} E\left(D_{N}^{a}\right)\right\}+\frac{1}{T_{C H}}\left\{\sum_{n=1}^{C H} E\left(D_{C H}^{n}\right)\right\}
\end{aligned}
$$

\subsubsection{Distance Computation}

Equation 9 demonstrates mathematically the fitness function of the distance between senor nodes to IoT base station. In order to choose the best $\mathrm{CH}$ distance between node to IoT base station should be minimum.

$F F_{\text {Distance }}=\sum_{a=1}^{X} \sum_{n=1}^{T_{c h}} \frac{\left\|D i s_{N}^{a}-D i s_{c h}^{n}\right\|+\left\|D i s_{c h}^{n}-D i s_{B S}\right\|}{M^{*} N}$

$\left\|D i s_{N}^{a}-D i s_{c h}^{n}\right\|$ is distance of $\mathrm{a}^{\text {th }}$ normal node from $\mathrm{n}^{\text {th }}$ cluster head.

$\left\|D i s_{c h}^{n}-D i s_{B S}\right\| i s$ distance between $\mathrm{n}^{\text {th }}$ cluster head to IoT base station. The denominator $\mathrm{M}$, $\mathrm{N}$ represents the dimension range in meters.

3.1.3 Delay Computation 
To select the best $\mathrm{CH}$, the delay should be less. The delay should be within the $[0,1]$ range. As the delay is dependent on nodes present in a cluster, the nodes present within a cluster should be minimal to minimize the delay. The fitness function of the delay transmission between IoT devices and $\mathrm{CH}$ is shown mathematically in Equation 10. The numerator represents the maximum transfer of data to the $\mathrm{BT}$ from a $\mathrm{CH}$ and the denominator represents the total number of nodes.

$$
F F_{\text {Delay }}=\frac{\operatorname{Max} \sum_{n=1}^{T_{c h}} c h_{n}}{X}
$$

\subsection{Temperature and Load Computation}

The temperature and load of the IoT devices are taken from the Xively IoT platform https://www.xively.com/. They should be low in order to improve the network life.

\subsection{WOA Algorithm}

WOA is one of the metaheuristic nature-inspired algorithm [35]. This algorithm was introduced based upon the hunting patterns of humpback whales. The attacking pattern of whales towards prey is called the bubble-net feeding mechanism which is done when bubbles are created in a circle shape around the prey. This mechanism composes of two phases Encircling Prey, Attacking Prey. The hunting behavior of whales is unique which has two techniques one is upward-spirals where the whales dive $12 \mathrm{~m}$ down the water and start creating bubbles around prey in a spiral shape. The second technique has three different stages loop capture, lobtail, and loop coral.

\subsubsection{Encircling Prey:}

The humpback whale tries to identify the position of prey and encircles prey. The whale assumes the current best solution is targeted prey and informs other search whales to change their position in the direction of the targeted prey. The encircling prey mechanism is represented in Equation [11], Equation [12].

$$
\begin{aligned}
& L=\left|\boldsymbol{Y} . S^{*}(k)-S(k)\right| \\
& S(k+1)=S^{*}(k)-X . L
\end{aligned}
$$

Where $\mathrm{X}, \mathrm{Y}$ are coefficient vectors, $\mathrm{k}$ stands for current iteration, $\mathrm{S} *$ is position vector of best solution, $\mathrm{S}$ is location of vector. The coefficient vectors $\mathrm{X}, \mathrm{Y}$ are shown in Equation [13], Equation [14]. Where the value of $\mathrm{x}$ decreases gradually from 2 to $0, \alpha$ lies in range of $[0,1]$. Figure 2 illustrates $\mathrm{S}^{*}$ of best solution.

$$
\begin{aligned}
& X=2 \boldsymbol{x} \propto-\boldsymbol{x} \\
& \boldsymbol{Y}=2 . \boldsymbol{\delta}
\end{aligned}
$$




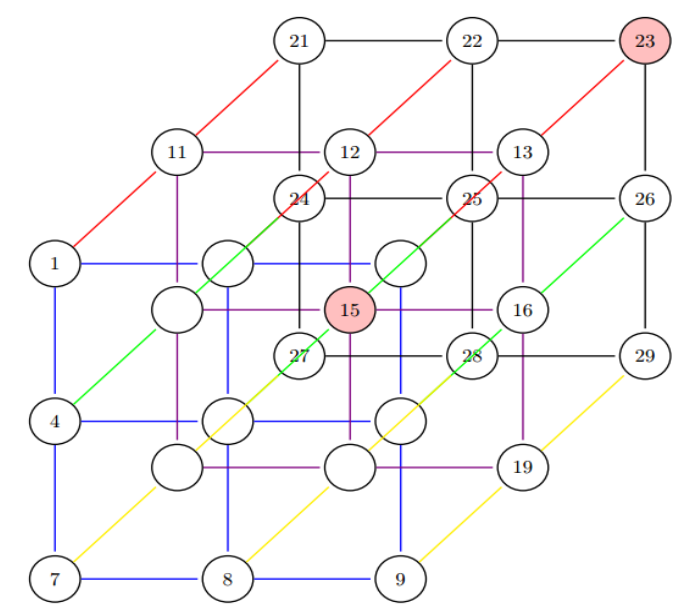

Figure 2: Position Vector of Best Solution

\section{1.2 Bubble-Net Attacking (Exploitation Phase)}

Humpback whale bubble-net attacking comprises of 2 approaches. The first approach is the shrinking encircling approach $\&$ spiral updating. To attack prey humpback whale uses any of these two techniques. The probability to catch prey using these approaches is around $50 \%$.

3.1.2.1 Shrinking Encircling Approach: Here, the value of ' $x$ ' starts decreasing from 2 to 0 which effects the value of $X$ given in Equation [13]. $X$ is a random number in the range of $[-\mathrm{X}, \mathrm{x}]$. The random values for $\mathrm{X}$ can be in the range of $[-1,1]$, which is illustrated in Figure $[3]$.

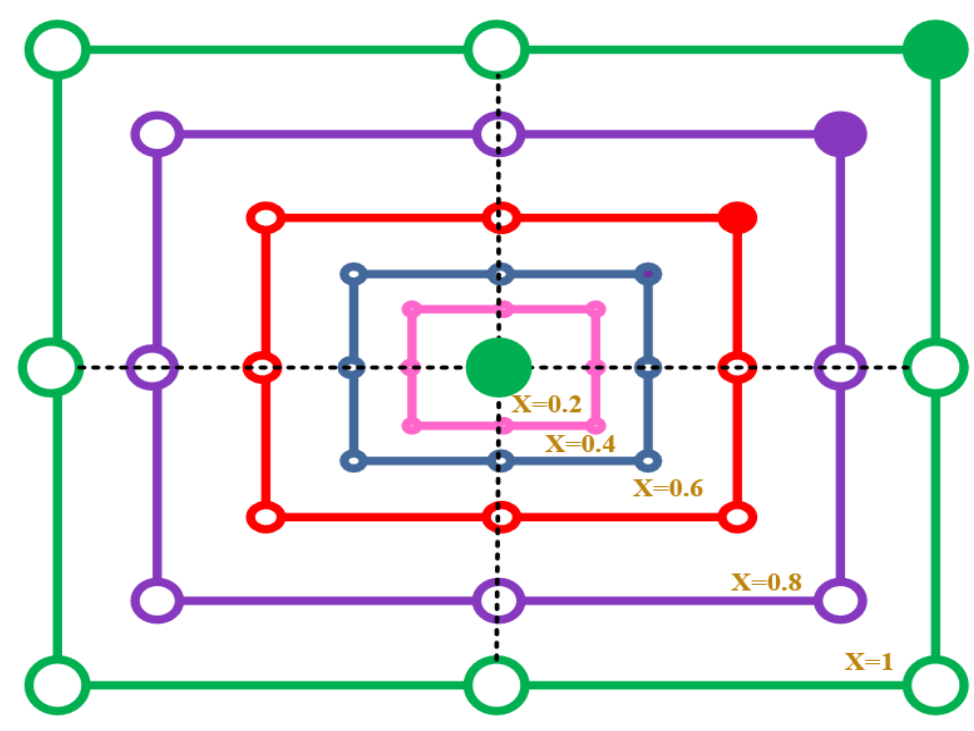

Figure 3: Possible best solutions

3.1.2.2 Spiral Updating Approach: In this approach, the distance of the whale towards the targeted prey is calculated using Equation [15].

$\boldsymbol{S}(\boldsymbol{k}+1)=\boldsymbol{L} \cdot \boldsymbol{e}^{m \boldsymbol{n}} \cdot \cos (2 \pi \boldsymbol{h})+\boldsymbol{S}^{*}(\boldsymbol{k})$ 
Where, $\boldsymbol{L}=\left|\boldsymbol{S}^{*}(\boldsymbol{k})-\boldsymbol{S}(\boldsymbol{k})\right|$ the distance of $\mathrm{i}^{\text {th }}$ whale and the targeted prey, which is the best position, $\mathrm{m}$ is a constant which defines the logarithmic spiral shape and $\mathrm{n}$ lies in the range [$1,1]$. Once the humpback whale identifies prey it swims around prey either in spiral-shape or shrinking circle. The probability of choosing either a spiral-shape or shrinking circle is $50 \%$. Updating the position of whales towards the best solution is shown in Equation [16].

$\boldsymbol{S}(\boldsymbol{k}+1)=\left\{\begin{array}{c}S(\boldsymbol{k})-X . L \text { if } P<0.5 \\ L . \boldsymbol{e}^{\boldsymbol{m} \boldsymbol{n}} \cdot \cos (2 \boldsymbol{\pi} \boldsymbol{h})+\boldsymbol{S}^{*}(\boldsymbol{k}) \text { if } P \geq 0.5\end{array}\right.$

\section{1.3 Searching of Prey (Exploration Phase):}

In this phase, the whale (search agent) globally searches for the best solution (prey) randomly and changes its position with respect to other whales. In order to force the search agent, move far towards other reference whales globally, the value of $\mathrm{X}$ should be greater than 1 or $\mathrm{X}$ should be less than -1. Searching of prey randomly is expressed in Equation [17], Equation [18]. Algorithm 1 explains WOA.

$$
\begin{aligned}
& \boldsymbol{L}=\left|\boldsymbol{Y} . \boldsymbol{S}_{\text {rand }}-\boldsymbol{S}\right| \\
& \boldsymbol{S}(\boldsymbol{k}+1)=\boldsymbol{S}_{\text {rand }}-\boldsymbol{X} \boldsymbol{L}
\end{aligned}
$$

\begin{tabular}{|c|c|}
\hline \multicolumn{2}{|c|}{$\begin{array}{l}\text { ALGORITHM 1: Pseudocode of WOA based cluster } \\
\text { head selection }\end{array}$} \\
\hline \multicolumn{2}{|c|}{ Initiate whales population $\mathrm{Si}=1,2, \ldots \ldots \mathrm{n}$} \\
\hline \multicolumn{2}{|c|}{ Compute the fitness of all agents } \\
\hline \multicolumn{2}{|c|}{ Allocate $\mathrm{S}^{*}$ as the best search agent } \\
\hline \multicolumn{2}{|c|}{ while $(\mathrm{k}<\mathrm{kMAX})$} \\
\hline \multicolumn{2}{|c|}{ for each search agent } \\
\hline \multicolumn{2}{|c|}{ Update $\mathrm{x}, \mathrm{X}, \mathrm{Y}, \mathrm{h}, \mathrm{P}$} \\
\hline \multicolumn{2}{|c|}{ if $(\mathrm{P}<0.5)$} \\
\hline \multicolumn{2}{|c|}{ if $(|X|<1)$} \\
\hline \multicolumn{2}{|r|}{$\begin{array}{l}\text { Update the current search agent } \\
\text { position using Eq. (11) }\end{array}$} \\
\hline \multicolumn{2}{|c|}{ else if $(|X| \geq 1)$} \\
\hline \multicolumn{2}{|r|}{ Choose a search agent $\left(\mathrm{S}_{\text {rand }}\right)$} \\
\hline \multicolumn{2}{|r|}{$\begin{array}{l}\text { Update the location of the search agent } \\
\text { using Eq. (18) }\end{array}$} \\
\hline \multicolumn{2}{|c|}{ end if } \\
\hline \multicolumn{2}{|c|}{ else if $(\mathrm{P}>=0.5)$} \\
\hline & $\begin{array}{l}\text { Update the location of the search agent } \\
\text { using Eq. (15) }\end{array}$ \\
\hline \multicolumn{2}{|l|}{ end if } \\
\hline end for & \\
\hline
\end{tabular}




\begin{tabular}{|l|l|}
\hline & $\begin{array}{l}\text { Evaluate the fitness of every search agent and } \\
\text { update } \mathrm{S}^{*} \text { if there is an optimal solution }\end{array}$ \\
\hline & Compute the fitness of each search agent \\
\hline $\begin{array}{l}\text { Update } \mathrm{S}^{*} \text { for the condition of having a better } \\
\text { solution }\end{array}$ \\
\hline $\mathrm{k}=\mathrm{k}+1$ \\
\hline End while \\
\hline Return $\mathrm{S}^{*}$ \\
\hline
\end{tabular}

Algorithm 1: Whale Optimization Algorithm

\subsection{Simulated Annealing}

Simulated Annealing (SA) is one of the optimization technique that is based on a concept in thermodynamics, especially the way metal will cool and then anneal [36]. SA is used to find the best global optima among several local optima. This algorithm uses an objective function of a corresponding optimization problem. This optimization algorithm is analogous to the hill-climbing technique. In the hill-climbing technique, the best move will be chosen for the objective function, whereas in simulated annealing, a random move will be chosen. If the chosen random move improves the solution, it will be accepted. Else, the algorithm makes a move with a random probability of less than 1 . This probability is given in the equation below:

Prob(uphill_move) 1-exp(deltaE/kT))

The parameter ' $\mathrm{T}$ ' is based on the temperature in the annealing problem in thermodynamics, which helps in finding the probability. Generally, ' $\mathrm{T}$ ' starts with a high value and with every step it keeps on decreasing. ' $\mathrm{k}$ ' is a parameter that correlates temperature to energy. In thermodynamics, it is analogous to Boltzmann's constant. SA can be used in very large and discrete search spaces like routing in VLSI, Travelling Salesman Problem, etc.

\section{Hybrid WSO-SA approach for cluster head selection process in IoT network}

The architecture of the proposed WSO-SA approach for the cluster head selection process in the IoT network is depicted in Figure [4]. As shown in Figure [4], the simulated IoT network generated consists of one base station (BT) with a number of clusters. In each cluster, many nodes, along with a cluster head, will be present. Each node transfers the data to the $\mathrm{CH}$. The $\mathrm{CH}$ will consolidate the data collected from all the nodes and send the same to the IoT BT. To select the best node for $\mathrm{CH}$, the hybrid WOA-SA approach is used [37]. The performance metrics considered for choosing the $\mathrm{CH}$ are a number of load, alive nodes, cost function, energy, and temperature. The algorithm for the proposed approach is discussed below: 


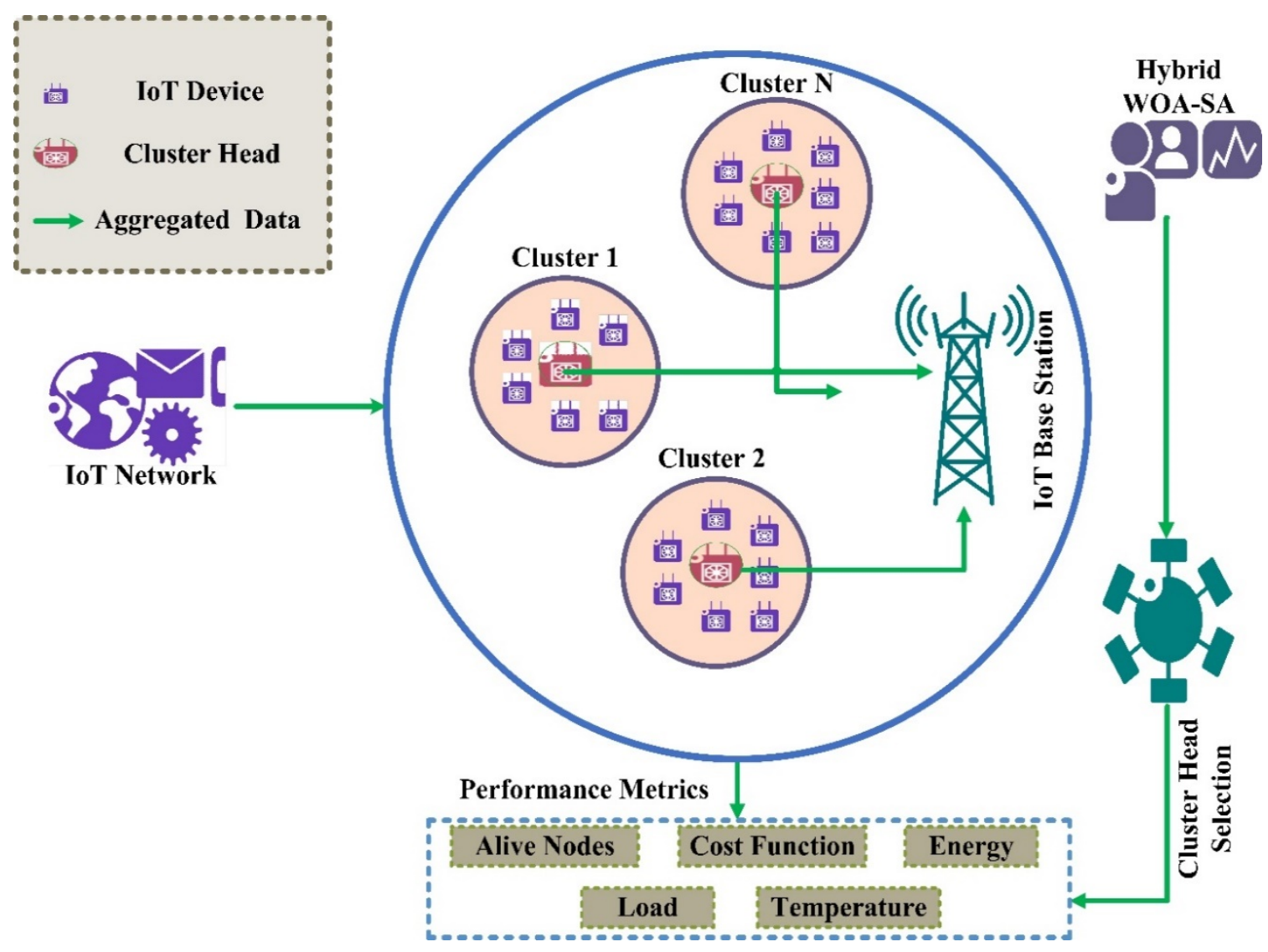

Figure 4: Schematic Representation of the Proposed Approach

\section{Results and Discussion}

The simulation of the cluster head selection in IoT is done in MATLAB R2015a. For this simulation, the data from the Xively IoT platform is used. In this work, to choose the best cluster head several performance metrics such as temperature, numbers of alive nodes, load, energy, cost function have been considered. The area considered for the simulation is $100 \mathrm{~m}$ $\mathrm{X} 100 \mathrm{~m}$. the IoT Base Station is assumed to be at the center of the area considered. The rest of this section discusses performance analysis of the proposed model with existing algorithms like the Artificial Bee Colony algorithm (ABC), Gravitational Search algorithm (GSO), Adaptive Gravitation Search algorithm (AGSA).

\section{1 Performance evaluation based on temperature metric}

Figure [5] demonstrates the performance of the proposed model with respect to the 'temperature' metric. Initially, the temperature on the sensor nodes is observed to be less for all the approaches. As the number of iterations increase, the temperature generated is also increased for all approaches. From Figure [5] it is observed that initially for all the approaches, the temperature generated is almost similar. But, after 1800 iterations, the temperature generated by using the proposed approach is comparatively less when compared to the existing approaches. 


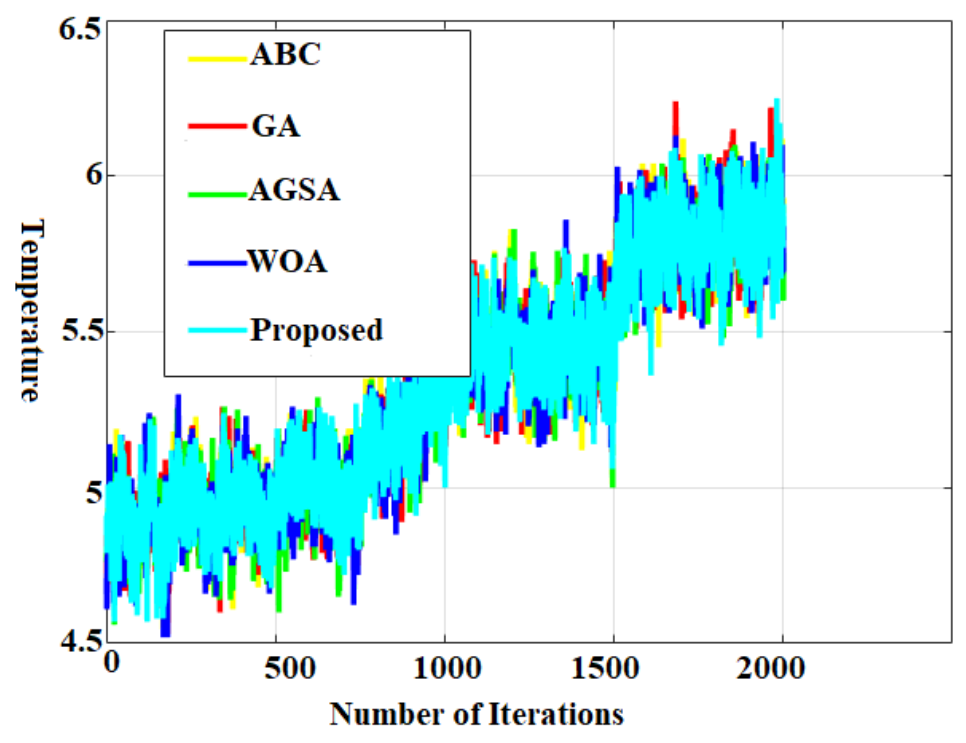

Figure [5]. Performance evaluation with respect to Temperature metric

\section{2 Performance evaluation based on the number of alive nodes}

The performance of the proposed model when compared to existing models based on the number of alive nodes is shown in Figure [6]. From this figure, it can be observed that during simulation, for the first 1000 iterations, all the nodes are alive. After 1000 iterations, the number of alive nodes started to decrease gradually for all the models. After 1700 iterations, there are no alive nodes for existing models. But the proposed model had around 20 alive nodes even after 1700 iterations. Hence the proposed model increases the lifetime of the network by keeping more nodes alive till the final iteration.

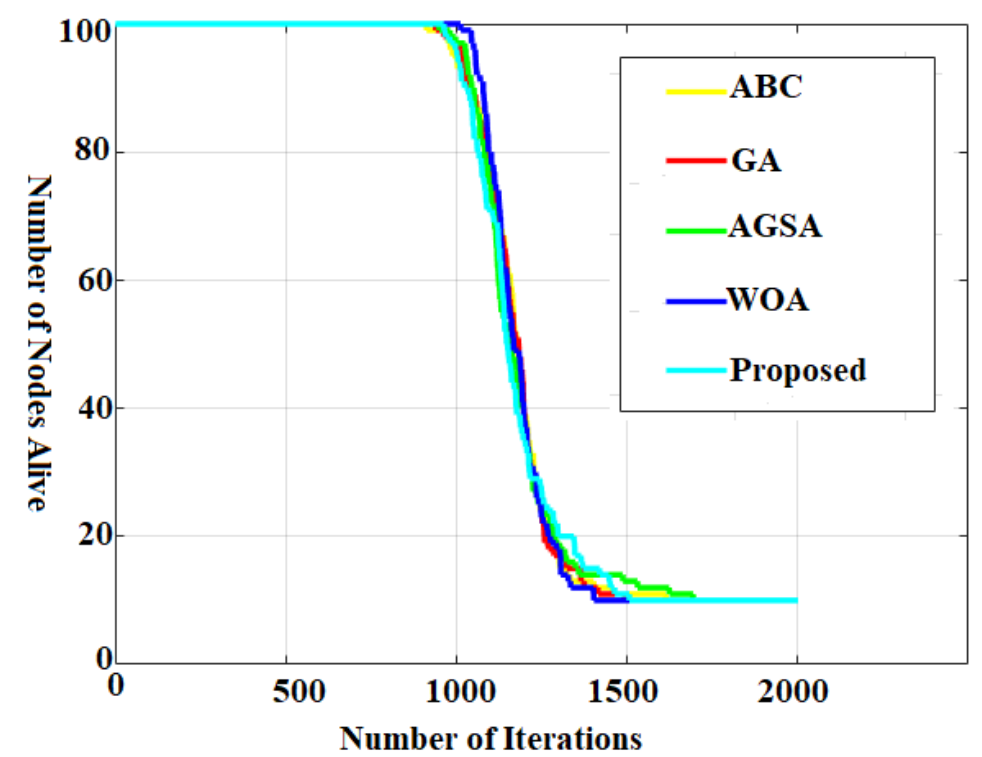

Figure [6]. Performance evaluation based on the number of alive nodes 


\subsection{Performance evaluation based on the Load metric}

Figure [7] demonstrates the performance of the proposed model based on the load of cluster heads. If the load of the IoT network is equally distributed among all the CHs, the performance of the network will be optimal. From this figure, it is observed that the proposed model distributes the load equally to all CHs. Also, the proposed model optimizes the load in all the iterations. The load of CHs is minimal even at 1500 iterations when compared with existing models. When the load is less on the $\mathrm{CH}$, the temperature generated by the sensor nodes will be less. This results in less energy consumption by the IoT network, which in turn improves the performance of the network.

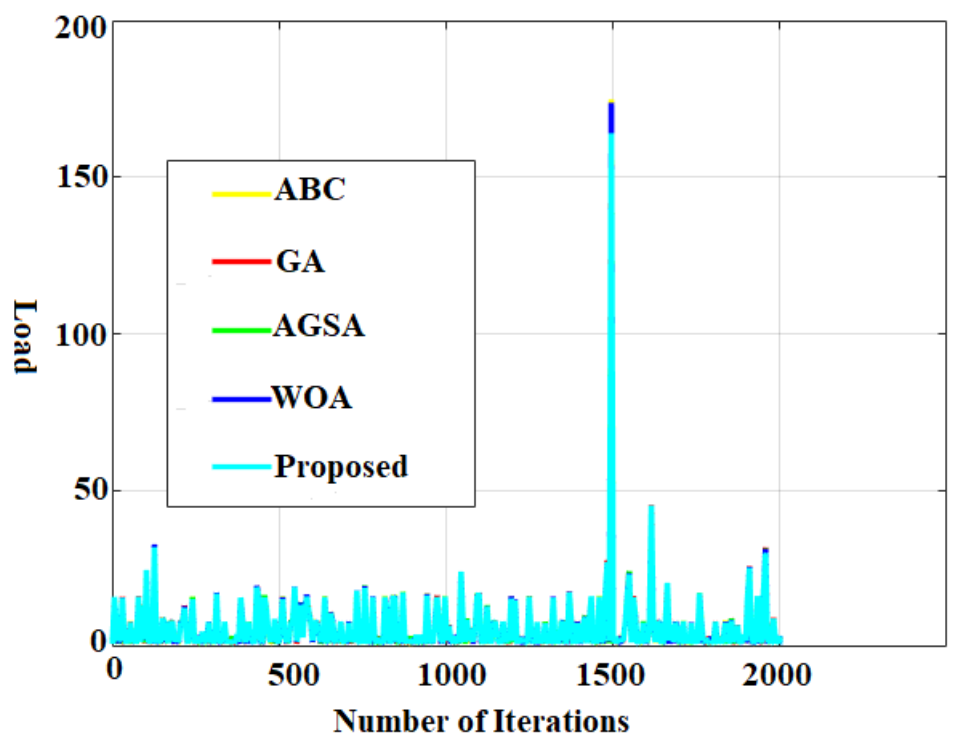

Figure [7]. Performance evaluation based on a load of CHs

\subsection{Performance evaluation based on the energy metric}

Figure [8] shows the performance of the proposed model based on energy when compared with other existing models. Initially, the energy of the network assumed for all models is 0.5 J. As the number of iterations increases, the energy starts to decrease. From the figure, it can be observed that compared to existing models, the proposed model had more energy in the network after all the iterations. This will help in increasing the sustainability of the network. 


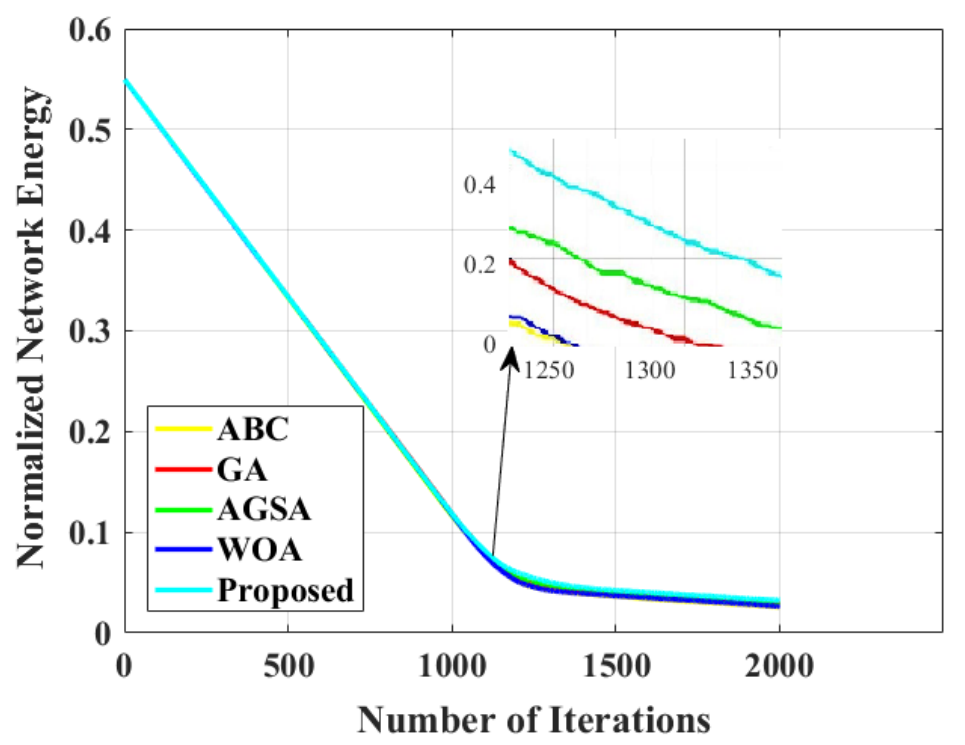

Figure [8]. Performance evaluation based on energy metric

\subsection{Performance evaluation based on the cost function}

Figure [9], demonstrates the performance of the proposed model based on the cost function. Cost function determines the convergence of an algorithm. Usually, as the number of iterations increases, the convergence of algorithms should be improved. From the figure, it can be observed that the convergence of the proposed model outperforms the existing models.

From the above figures, [5] to [9], it is clear that the proposed model outperforms the existing models with respect to all the measures considered. As the existing models use the blind operator for exploitation purposes irrespective of fitness functions. In the WOA-SA method, simulated annealing, which acts as an operator, ensures that the blind operator is replaced with a local search consisting of the solution itself as an initial state. Later, after working on the local search, the enhanced solution replaces the original solution. Thus, the simulated annealing process enhances the exploitation capacity of the WOA algorithm. Hence the simulated annealing algorithm helps in improving the performance of the whale optimization algorithm to find the optimal solution. Thus, the proposed model has an advantage over the existing models in optimizing the performance of the IoT network. 


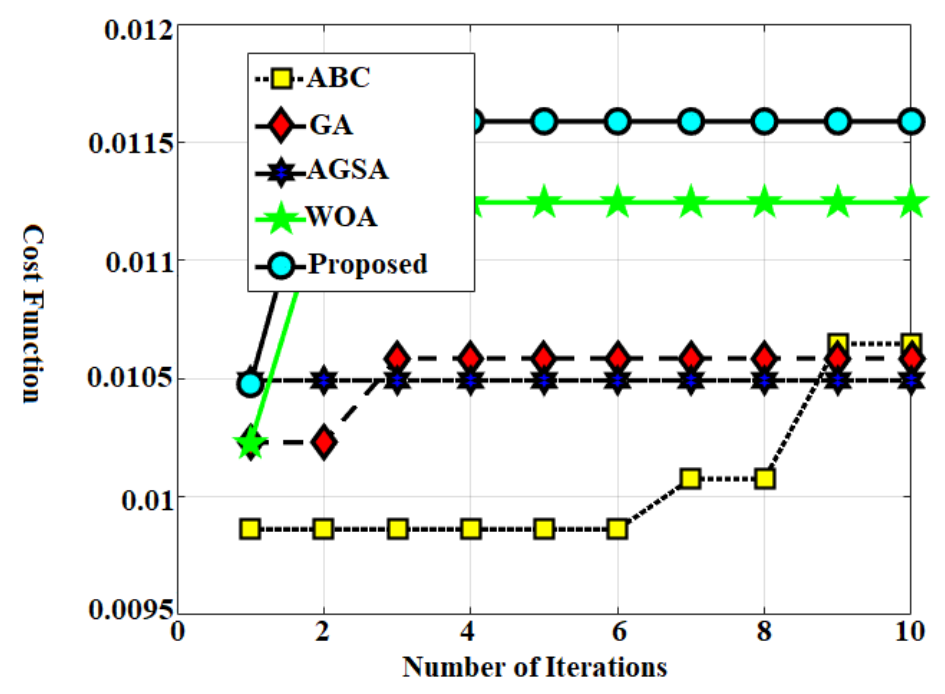

Figure [9]. Performance evaluation based on cost function.

\section{Conclusion and Future Work}

Even though IoT has enormous potential in several applications in the present era, there exist several challenges. Some of the challenges that need to be addressed to enhance the robustness of IoT are security, energy optimization, routing, hardware compatibility issues, data connectivity issues, etc. In the present work, we chose to address the energy optimization issue. To address this issue, in the present work a hybrid meta-heuristic algorithm, namely, Whale Optimization Algorithm-Simulated Annealing, is used to optimize the energy consumption of the sensors in IoT based Wireless Sensor Networks. To simulate the IoT network, the Xively IoT platform is used in this work. The IoT network has been run for 2000 iterations. Several performance metrics like load, residual energy, number of alive nodes, cost function, the temperature is used in this work to choose the optimal cluster heads in the IoT network. The proposed approach is then compared with several state-of-art approaches. The experimentation results prove that the proposed approach outperforms the existing approaches. In the future, to further optimize the energy utilization, several other performance metrics like delay, node density, link lifetime, etc. can be considered to choose the optimal cluster head. Also, the proposed work can be tested for scalability by using this approach in real-time applications like those applied in [38-43] with a huge number of sensors. Also, in this work, the energy consumption of the entire IoT network is evaluated. In future works, energy optimization in every individual cluster in IoT network can be studied; security issues solved by several researchers [44-47] can be applied for the proposed model to address the security issues.

\section{COMPETING INTERESTS}

The authors announce that there is no discrepancy of interests concerning the publication of this research.

\section{References}

1. Verma, S., Kawamoto, Y., Fadlullah, Z. M., Nishiyama, H., \& Kato, N. (2017). A survey on network methodologies for real-time analytics of massive IoT data and open research issues. IEEE Communications Surveys \& Tutorials, 19(3), 1457-1477.

2. Miorandi, D., Sicari, S., De Pellegrini, F., \& Chlamtac, I. (2012). Internet of things: Vision, applications and research challenges. Ad hoc networks, 10(7), 1497-1516. 
3. Čolaković, A., \& Hadžialić, M. (2018). Internet of Things (IoT): A review of enabling technologies, challenges, and open research issues. Computer Networks, 144, 17-39.

4. Ding, X., \& Wu, J. (2019). Study on Energy Consumption Optimization Scheduling for Internet of Things. IEEE Access.

5. Praveen Kumar Reddy, M., \& Rajasekhara Babu, M. (2017). Energy efficient cluster head selection for internet of things. New Review of Information Networking, 22(1), 54-70.

6. S. K. Sathya Lakshmi Preetha, R. Dhanalakshmi, and R. Kumar, "An Energy Efficient Framework for Densely Distributed WSNs IoT Devices Based on Tree Based Robust Cluster Head", Wireless Personal Communications, vol. 103, no. 4, pp 3163-3180, 2018.

7. Jine Tang, ZhangBing Zhou, Jianwei Niu, and Qun Wang. An energy efficient hierarchical clustering index tree for facilitating time-correlated region queries in the internet of things. Journal of Network and Computer Applications, 40:1-11, 2014.

8. Qingjian Ni, Qianqian Pan, Huimin Du, Cen Cao, and Yuqing Zhai. A novel cluster head selection algorithm based on fuzzy clustering and particle swarm optimization. IEEE/ACM transactions on computational biology and bioinformatics, 14(1):76-84, 2015.

9. Song, L., Chai, K. K., Chen, Y., Loo, J., Jimaa, S., \& Iraqi, Y. (2019). Energy efficient cooperative coalition selection in cluster-based capillary networks for CMIMO IoT systems. Computer Networks, 153, 92-102.

10. Thangaramya, K., Kulothungan, K., Logambigai, R., Selvi, M., Ganapathy, S., \& Kannan, A. (2019). Energy aware cluster and neuro-fuzzy based routing algorithm for wireless sensor networks in IoT. Computer Networks, 151, 211-223.

11. Jesudurai, S. A., \& Senthilkumar, A. (2019). An improved energy efficient cluster head selection protocol using the double cluster heads and data fusion methods for IoT applications. Cognitive Systems Research, 57, 101-106.

12. Reddy, M. P. K., \& Babu, M. R. (2019). A hybrid cluster head selection model for Internet of Things. Cluster Computing, 22(6), 13095-13107.

13. Reddy, M. P. K., \& Babu, M. R. (2019). Implementing self adaptiveness in whale optimization for cluster head section in Internet of Things. Cluster Computing, 22(1), 1361-1372.

14. Dhumane, A. V., \& Prasad, R. S. (2019). Multi-objective fractional gravitational search algorithm for energy efficient routing in IoT. Wireless networks, 25(1), 399413.

15. Srinidhi, N. N., Lakshmi, J., \& Kumar, S. D. (2019, February). Hybrid Energy Efficient and QoS Aware Algorithm to Prolong IoT Network Lifetime. In International Conference on Ubiquitous Communications and Network Computing (pp. 80-95). Springer, Cham.

16. Feng, X., Zhang, J., Ren, C., \& Guan, T. (2018). An unequal clustering algorithm concerned with time-delay for Internet of Things. IEEE Access, 6, 33895-33909.

17. Lin, K., Wang, D., Xia, F., \& Ge, H. (2017). Device clustering algorithm based on multimodal data correlation in cognitive Internet of Things. IEEE Internet of Things Journal, 5(4), 2263-2271.

18. Puschmann, D., Barnaghi, P., \& Tafazolli, R. (2016). Adaptive clustering for dynamic IoT data streams. IEEE Internet of Things Journal, 4(1), 64-74.

19. Shao, X., Yang, C., Chen, D., Zhao, N., \& Yu, F. R. (2018). Dynamic IoT device clustering and energy management with hybrid NOMA systems. IEEE Transactions on Industrial Informatics, 14(10), 4622-4630. 
20. Xiong, J., Ren, J., Chen, L., Yao, Z., Lin, M., Wu, D., \& Niu, B. (2018). Enhancing privacy and availability for data clustering in intelligent electrical service of IoT. IEEE Internet of Things Journal, 6(2), 1530-1540.

21. Sadek, R. A. (2018). Hybrid energy aware clustered protocol for IoT heterogeneous network. Future Computing and Informatics Journal, 3(2), 166-177.

22. Alvi, A. N., Khan, S., Javed, M. A., Konstantin, K., Almagrabi, A. O., Bashir, A. K., \& Nawaz, R. (2019). OGMAD: Optimal GTS-allocation mechanism for adaptive data requirements in IEEE 802.15. 4 based Internet of Things. IEEE Access.

23. Dar, Z., Ahmad, A., Khan, F. A., Zeshan, F., Iqbal, R., Sherazi, H. H. R., \& Bashir, A. K. (2019). A context-aware encryption protocol suite for edge computing-based IoT devices. The Journal of Supercomputing, 1-20.

24. Iwendi, C., Alqarni, M. A., Anajemba, J. H., Alfakeeh, A. S., Zhang, Z., \& Bashir, A. K. (2019). Robust navigational control of a two-wheeled self-balancing robot in a sensed environment. IEEE Access, 7, 82337-82348.

25. Mittal, M., \& Iwendi, C. (2019). A Survey on Energy-Aware Wireless Sensor Routing Protocols. EAI Endorsed Transactions on Energy Web, 6(24).

26. Mittal, M., Saraswat, L. K., Iwendi, C., \& Anajemba, J. H. (2019, April). A NeuroFuzzy Approach for Intrusion Detection in Energy Efficient Sensor Routing. In 2019 4th International Conference on Internet of Things: Smart Innovation and Usages (IoT-SIU) (pp. 1-5). IEEE.

27. Thippa Reddy, G., Sudheer, K., Rajesh, K., \& Lakshmanna, K. (2014). Employing data mining on highly secured private clouds for implementing a security-asa-service framework. Journal of Theoretical and Applied Information Technology, 59(2), 317326.

28. Raghavan, R, Singh, J.K, Reddy, T.G, Sudheer, K, Venkatesh, P, Olabiyisi, S.O, (2017). A case study: Home environment monitoring system using internet of things, International Journal of Mechanical Engineering and Technology. 8 (11), 173-180.

29. Reddy, G. T., Kaluri, R., Reddy, P. K., Lakshmanna, K., Koppu, S., \& Rajput, D. S. (2019). A Novel Approach for Home Surveillance System Using IoT Adaptive Security. Available at SSRN 3356525.

30. Mittal, M. and Iwendi, C., A Survey on Energy-Aware Wireless Sensor Routing Protocols EAI Endorsed Transactions on Energy Web, Year: 2019

31. Iwendi, C., Alastair, A., Offor, K., Smart Security Implementation for Wireless Sensor Network Nodes. Journal of Wireless Sensor Network, [S.1.], v. 1, aug. 2015. ISSN 2001-6417.

32. Mittal, M., Saraswat, L. K., Iwendi, C., and Anajemba, J. H., A Neuro-Fuzzy Approach for Intrusion Detection in Energy Efficient Sensor Routing, 2019 4th International Conference on Internet of Things: Smart Innovation and Usages (IoTSIU), Ghaziabad, India, 2019, pp. 1-5.

33. Iwendi, C., Zhang, A., and Du, X., ACO based key management routing mechanism for WSN security and data collection, 2018 IEEE International Conference on Industrial Technology (ICIT), Lyon, France, 2018, pp. 1935-1939.

34. Iwendi, C.O.; Allen, A.R.; Enhanced security technique for wireless sensor network nodes, Wireless Sensor Systems (WSS 2012), IET Conference on, vol., no., pp.1-5, 18-19 June 2012

35. Mirjalili, S., \& Lewis, A. (2016). The whale optimization algorithm. Advances in engineering software, 95, 51-67.

36. Zhang, W., Maleki, A., Rosen, M. A., \& Liu, J. (2018). Optimization with a simulated annealing algorithm of a hybrid system for renewable energy including battery and hydrogen storage. Energy, 163, 191-207. 
37. Mafarja, M. M., \& Mirjalili, S. (2017). Hybrid whale optimization algorithm with simulated annealing for feature selection. Neurocomputing, 260, 302-312.

38. Iwendi, C., Suresh, P., Revathi, M., Srinivasan, K., and Chuan-Yu Chang, An Efficient and Unique TF/IDF Algorithmic Model-Based Data Analysis for Handling Applications with Big Data Streaming, published in Artificial IntelligenceApplications and Methodologies of Artificial Intelligence in Big Data Analysis in Electronics 2019,8(11),1331

39. Iwendi, C., Uddin, M., Ansere, J. A., Nkurunziza, P., Anajemba, J. H. and Bashir, A. K., On Detection of Sybil Attack in Large-Scale VANETs Using Spider-Monkey Technique, in IEEE Access, vol. 6, pp. 47258-47267, 2018.

40. Iwendi, C., Alqarni, M. A., Anajemba, J. H., Alfakeeh, A. S., Zhang,. Z and Bashir, A. K., Robust Navigational Control of a Two-Wheeled Self-Balancing Robot in a Sensed Environment, in IEEE Access, vol. 7, pp. 82337-82348, 2019.

41. Kutia, S., Chauhdary, S. H., Iwendi, C., Liu, L., Yong, W., and Bashir, A. K., SocioTechnological Factors Affecting User's Adoption of eHealth Functionalities: A Case Study of China and Ukraine eHealth Systems, in IEEE Access, vol. 7, pp. 9077790788, 2019.

42. Vincent, D.R.; Deepa, N.; Elavarasan, D.; Srinivasan, K.; Chauhdary, S.H.; Iwendi, C. Sensors Driven AI-Based Agriculture Recommendation Model for Assessing Land Suitability. Sensors 2019, 19, 3667.

43. S.J. Lim; A.K. Bashir; S.Y. Rhee; and M.S. Park. Energy-Based Re-transmission Algorithm of the Leader Node's Neighbor Node for Reliable Transmission in the PEGASIS. Computing and Multimedia Applications. UCMA 2011. CCIS, vol. 150. Springer, Berlin, Heidelberg. A book chapter.

44. Chaudhary, R., Aujla, G. S., Garg, S., Kumar, N., \& Rodrigues, J. J. (2018). SDNenabled multi-attribute-based secure communication for smart grid in IIoT environment. IEEE Transactions on Industrial Informatics, 14(6), 2629-2640.

45. He, D., Kumar, N., Khan, M. K., Wang, L., \& Shen, J. (2016). Efficient privacyaware authentication scheme for mobile cloud computing services. IEEE Systems Journal, 12(2), 1621-1631.

46. Wazid, M., Das, A. K., Odelu, V., Kumar, N., \& Susilo, W. (2017). Secure remote user authenticated key establishment protocol for smart home environment. IEEE Transactions on Dependable and Secure Computing.

47. Gope, P., Amin, R., Islam, S. H., Kumar, N., \& Bhalla, V. K. (2018). Lightweight and privacy-preserving RFID authentication scheme for distributed IoT infrastructure with secure localization services for smart city environment. Future Generation Computer Systems, 83, 629-637. 